\title{
Improving surgical weekend handover
}

\author{
Caroline Culwick, Chris Devine, Catherine Coombs \\ GWH
}

\begin{abstract}
Effective handovers are vital to patient safety and continuity of care, and this is recognised by several national bodies including the GMC. The existing model at Great Western Hospital $(\mathrm{GWH})$ involved three general surgical teams and a urology team placing their printed patient lists, complete with weekend jobs, in a folder for the on-call team to collect at the weekend. We recognised a need to reduce time searching for patients, jobs and reviews, and to streamline weekend ward rounds.

A unified weekend list ordering all surgical patients by ward and bed number was introduced. Discrepancies in the layout of each team's weekday list necessitated the design of a new weekday list to match the weekend list to facilitate the easy transfer of information between the two lists. A colour coding system was also used to highlight specific jobs.

Prior to this improvement project only $7.1 \%$ of those polled were satisfied with the existing system, after a series of interventions satisfaction increased to $85.7 \%$. The significant increase in overall satisfaction with surgical handover following the introduction of the unified weekend list is promising. Locating patients and identifying jobs is easier and weekend ward rounds can conducted in a more logical and timely fashion. It has also helped facilitate the transition to consultant ward rounds of all surgical inpatients at the weekends with promising feedback from a recent consultants meeting.
\end{abstract}

\section{Problem}

The current surgical weekend handover system at the Great Western Hospital in Swindon involves each of three general surgical teams and the urology team printing their patient lists and placing them in a folder for the on-call team. Patient reviews and jobs for the weekend are written on the list with an indication of which grade of doctor should be responsible. Each team has patients split across the 5 surgical wards.

Having four separate lists makes it difficult to identify patient reviews quickly and is considered by the majority of staff to be cumbersome and inefficient. A typical weekend consists of one registrar, one $\mathrm{SHO}$, one clerking $\mathrm{F} 1$ and two ward $\mathrm{F} 1 \mathrm{~s}$ responsible for all surgical patients. Senior surgeons have little time out of emergency theatre to review ward patients and there have been instances where ill patients have not been reviewed. Systematic ward rounds at the weekend are hampered by not having a single list of patients organised by ward and bed number. Furthermore, each team's list varies in its layout and information, leading to discrepancies in the quality and format of patient handover.

Time is wasted identifying patients for review or manually collating jobs from the separate lists. This has the potential to delay patient review or treatment and increases the chance of incomplete jobs. These problems compromise patient safety and create a stressful environment for the on-call team.

\section{Background}

Weekend on-call teams are stretched and unfamiliar with the patients under their care. To compound the problem routine jobs such as discharge summaries and rewriting drug charts are often left for the weekend team which further limits their time. Effective handover is vital to ensure continuation of high quality care. As there is no standardised national handover system there is a large variation in the quality of handover between hospitals.

The importance of clear weekend handover is one that is recognised by the GMC in parts 44 \& 45 of "Good Medical Practice" (1) and the BMA in "Safe Handover: Safe Patients" (2), while the Royal College of Surgeons identifies handover as the point at which patients are most vulnerable in their journey through the healthcare system (3). Trends in mortality show that admission to hospital towards the end of the week is associated with a higher risk compared with admission earlier in the week $(4,5)$.

Concerns about the safety and efficacy of in-hospital weekend handover are long-standing and have been addressed by junior staff at other hospitals in other quality improvement projects $(6,7,8,9,10,11,12)$. Systems that are entirely paper based have been identified as disorganised, lacking in information and often illegible $(6,7,9)$. Electronic systems on the other hand, with information available to the weekend team on a shared drive on any hospital computer, have been shown to improve handover and are crucially easier to audit $(9,10,11)$. It has been noted, however, that electronic handover systems may be difficult to implement unless commercial software is purchased and installed (11). Other strategies which have been trialled with mixed results include handover stickers $(8)$ or proformas $(6,7)$ in patients' notes. Both of these strategies improve the quality of information available to weekend teams but do not help with identifying patients in need of review or obtaining up to date patient location. 


\section{Baseline measurement}

Concerns about handover were discussed with other surgical juniors. Consensus was that the system was inefficient, frustrating and conducive to missing jobs.

Three baseline measures were undertaken:

A questionnaire was disseminated to quantify this using answers graded on a 1-5 scale with space for free text (see attached questionnaire). There were 14 respondents, which represents the majority of surgical juniors, and all had worked at least two weekends to this point.

Time wasted looking for patients on the handovers lists was raised as a concern. To quantify it the time taken to locate a patient when provided with their name, ward and bed number was measured with a stop watch.

A large number of surgical F1s raised the issue that they were so concerned about missing jobs using the existing system that they spent a significant amount of time in the mornings at the weekend manually collating the jobs that needed doing onto their own list of jobs. The average time spent doing this was collected from all of the F1s who had worked a weekend on surgery.

See supplementary file: ds3681.docx - "Weekend Handover Questionnaire"

\section{Design}

Two interventions were considered: a unified weekend list and weekend handover proformas. Initial discussion with other juniors indicated a preference for a unified list. It was felt that this would improve efficiency without over-burdening the week team with extra work on Fridays.

A unified weekend list ordered by ward and bed number was designed. Space was incorporated to indicate completion of discharge paperwork and the days to take bloods. These were two pieces of information identified as useful to the ward F1s. A blank copy of the list was uploaded to an intranet drive accessible to all surgical juniors.

The proposed new system involved each of the week teams copying their Friday list to the new weekend template. This would leave a complete list of all surgical patients ordered by ward and bed number ready for the on call team. A trial of this system for two weeks followed by review was agreed between the project team and junior surgical staff.

\section{Strategy}

PDSA cycle 1: Baseline measurement took place in the form of a questionnaire disseminated to surgical $\mathrm{F} 1 \mathrm{~s}$, SHOs and registrars. To quantify time saved by introducing the new list several volunteers from the surgical teams were recruited to identify time taken to locate patients on the existing lists from their name, ward and bed number. The volunteers were also asked to estimate how long they spent compiling a list of jobs on a Saturday morning when they worked the weekend. The new weekend list was based on recommendations from discussions with other surgical juniors. This list was then uploaded onto a shared network drive. All surgical F1s and $\mathrm{SHO}$ were informed that the project was taking place, shown how to access the list and how to copy information across to it. One member of the project team was available to answer questions and address any initial issues. When all the teams had copied across their patients the final list was saved on the saved drive and hard copies were left in the usual place in the surgical offices.

In PDSA cycle 1 the patients were listed by ward but not bed number and a paper copy was printed off by the project team on Friday afternoon in addition to the electronic file. Following two trial weekends formal and informal feedback was gained in the form of repeating the timed tests and holding a discussion at one of the surgical teaching sessions. All of those present agreed the new list improved upon the old system and a number of helpful suggestions were put forward including ordering the patients by bed number, combining some of the columns on the weekend list, getting the F1 covering the wards on Saturday to print off the list first thing in the morning to avoid missing any late additions to the list and for the weekend F1s to update the weekend list over the weekend with any major changes to patients.

PDSA cycle 2: Taking on board the feedback from the meeting the weekend list was modified slightly and the new copy uploaded onto the shared drive for continued use. The time taken to find patients under this system and compile jobs were measured again using this list format.

PDSA cycle 3: The major problem identified post cycle 2 was the difficulty in copying across information from the week lists to the weekend lists due to their varying formats. Those consulted estimated it was taking them 10-20 minutes to copy their patients onto the weekend list, which was considered unsatisfactory. To address this a standard weekday list was designed for each of the surgical teams based on the format of the new weekend list. Each team's patients were copied across from their old lists to their new standardised list by the project team. The teams were happy with the new lists and used them exclusively from the Tuesday after they were introduced. With the introduction of the new lists time taken to transfer the patients between the week and weekend lists reduced to less than 5 minutes per team. Another meeting with the surgical juniors was arranged to discuss a further intervention; the project team felt it was still relatively difficult to identify jobs and patients needing review on the lists.

PDSA cycle 4: Consideration was given to grouping the patients on each ward by registrar/SHO/F1 review. This would enable each grade of doctor to quickly identify which patients they needed to review. Informal feedback from surgical juniors at the meeting deemed this unpopular. There was preference to keep patients ordered by bed number within each ward on the list. After discussion it was felt a colour coding system would be helpful in highlighting relevant reviews for each grade of doctor. At the end of 
this intervention formal post improvement project data was collected in the form of the questionnaire used prior to commencing the project and the timings we had used throughout the project repeated one last time.

See supplementary file: ds3101.docx - "Weekend list versions 1 and 2"

\section{Post-measurement}

An identical questionnaire was dissemination before (Q1) and after (Q2) the completion of all PSDA cycles. There were fourteen respondents to each questionnaire. The nine questions were scored from 1 (very unsatisfied) to 5 (very satisfied).

The full results are detailed in the attached tables.

Table 9 illustrates that $7.1 \%$ of respondents in Q1 ranked overall satisfaction with the handover system as 4 or $5.85 .7 \%$ ranked the same question as 4 or 5 in Q2.

Table 1 refers to the question "How easy is it to know who needs a weekend review?". $50 \%$ of respondents in Q1 ranked this as 4 or 5 compared with $100 \%$ in Q2.

Table 2 refers to the question "How often is it written why patients need review?". None of the respondents in Q1 ranked this as 4 or 5 compared with $21.4 \%$ in Q2.

Table 3 refers to the question "How often do you know which day you need to review the patient?". 42.9\% of respondents in Q1 ranked this as 4 or 5 compared with $85.7 \%$ in Q2.

Table 4 refers to the question "How often do you feel you are reviewing patients unnecessarily?". In this question a score of 5 equates to "always" and a score of 1 equates to "never". $21.4 \%$ of respondents in Q1 ranked this as 4 or 5 compared with $14.3 \%$ in Q2.

Table 5 refers to the question "How often do you feel has not been reviewed who should have been?". In this question a score of 5 equates to "always" and a score of 1 equates to "never". $28.6 \%$ of respondents in Q1 ranked this as 4 or 5 compared with $14.3 \%$ in Q2.

Table 6 refers to the question "How often do patients have clear weekend plans?". $21.4 \%$ of respondents in Q1 ranked this as 4 or 5 compared with $28.6 \%$ in Q2.

Table 7 refers to the question "How often do you think unnecessary bloods are being done?". In this question a score of 5 equates to "always" and a score of 1 equates to "never". $57.1 \%$ of respondents ranked this as 4 or 5 in both Q1 and Q2.

Table 8 refers to the question "How often is it stated which day(s) patients need bloods checking?". $61.5 \%$ of respondents ranked this as 4 or 5 in Q1 compared with $100 \%$ in Q2.
The average time taken to locate a patient on the old style of lists was 21 seconds, compared to 15 seconds at the end of PDSA cycle 1 and 4 seconds with the final version of the weekend list. The difference in time between the original list and the final version is statistically significant even with the relatively small sample size used. (see Sheet 2 in Tables of results)

The reported average time spent compiling a list of jobs on a day at the weekend fell from 30-40 minutes pre-intervention to 13-15 minutes after PDSA cycle 1, 10-12 minutes at the end of PDSA cycle 2 and less than 5 minutes at the end of PDSA cycle 4 (table 10).

The reported average time spent copying information from the weekday lists to the weekend lists was 15-20 minutes before the introduction of the standardised week team lists (PDSA cycle 3) this fell to less than 5 minutes after their introduction (Table 11).

See supplementary file: ds3777.xlsx - "Tables of results"

\section{Lessons and limitations}

We learnt several lessons during the project. Firstly, it was important to engage all those who were involved with generating weekend plans in addition to those working the weekend. Secondly, the intervention had to be easy to adopt with benefits that were directly tangible. Thirdly, our intervention had to avoid burdening the weekday teams with excessive extra work.

The unified list was introduced on the weekend before Christmas and initial uptake was poor. In retrospect this may have been avoided by implementing the list after the New Year. However, after a few weeks there was universal and autonomous use of the list amongst the surgical teams. This showed a level of engagement with the project that has allowed it to succeed and continue to be used in the long term.

A benefit seen which was not a specific aim of the project is a general improvement in the content of the handover lists over the course of the project. This is an area that still has great scope for improvement and could be a potential focus of a follow-up project.

While we feel that there has been a small increase in the time taken to complete the new weekend list compared to the old system we believe that this has been made negligible by matching the format of the week team lists to the weekend sheet. It is hoped that the time saved by the on-call team vindicates this. It was valuable to collect informal verbal feedback as we went along allowing us to tweak a few aspects of the list to make it work more effectively.

There are two notable limitations to our study. Firstly the respondents in Q1 were not all the same respondents as in Q2. Unfortunately this was necessitated by the moving of staff between jobs during the December changeover. Secondly, given the intimate department size of this district general hospital, it is not possible to completely exclude personal bias in the results since the respondents are colleagues we work with. However it is hoped that the fact that the respondents differed in each questionnaire negates 
this somewhat, since there would have been no knowledge of the results of Q1 in those completing Q2.

\section{Conclusion}

The pre-existing surgical weekend handover system was archaic and inefficient. It was difficult to easily identify patients for review and carrying around five patient lists was awkward and clumsy. Our new unified weekend list contains every surgical patient in the hospital arranged by ward and bed number. Carrying one list is easier when on-call and provides a simple reference for identifying patients for review.

The increase in doctor satisfaction with the system from $7.1 \%$ to $85.7 \%$ is very encouraging. Informal feedback suggests that the weekend list has been enabling the on-call team to focus more time on patient reviews and jobs and this is supported by the finding that it was significantly quicker to find patients on the new weekend list. This has been notably valuable on weekends where large volumes of surgical admissions have led to surgical outliers on medical wards.

We believe patient safety has improved for two key reasons. Firstly, there is less likelihood of missed patient reviews, particularly on the newly-introduced weekend consultant ward rounds. Secondly, the time that has been saved by improving efficiency of handover can be directly reinvested into patient care.

A limitation of the project is that its scope did not include improving the quality of information handed over; we aimed to improve the system of handover. On reviewing a random selection of handover lists from the old and new systems we have informally observed a subjective improvement in the quality of information. It is possible that raising awareness of the shortcomings of the previous system of handover has precipitated this. Formally attempting to improve the quality of information handed over would be a logical follow on project from this one.

The project was discussed at a monthly surgical consultants' meeting as part of a wider discussion about the nationwide move to weekend consultant ward rounds. Feedback from this meeting was positive and there has been widespread approval from the consultant surgeons, both with the system of handover and the quality of information provided on the lists. It has helped streamline their weekend ward rounds and will hopefully be a useful resource for them.

\section{References}

1. General Medical Council. Good medical practice. London. General Medical Council. 2013

2. British Medical Association. Safe handover: safe patients. Guidance on clinical handover for clinicians and managers. London: BMA, 2004.

3. The Royal College of Surgeons of England. Safe handover: Guidance from the Working Time Directive working party. London: RCSE, 2007.
4. Dr Foster Hospital Guide (2011), Reducing mortality at nights and weekends, pg 19-22 (http://drfosterintelligence.co.uk/wpcontent/uploads/2011/11/Hospital Guide 2011.pdf)

5. Aylin P, Alexandrescu R, Jen MH, Mayer EK, Bottle A. Day of week of procedure and 30 day mortality for elective surgery: retrospective analysis of hospital episode statistics. BMJ. 2013 May 28;346:f2424. doi: 10.1136/bmj.f2424.

6. Din N, Ghaderi S, O'Connell R, Johnson T. Strengthening surgical handover: Developing and evaluating the effectiveness of a handover tool to improve patient safety. BMJ Quality Improvement Reports 2012; 1, No. 1 u492.w164 doi: 10.1136/bmjquality.u492.w164

7. Ashton C. Improving weekend patient handover. BMJ Quality Improvement Reports 2013; u201303.w827 doi: 10.1136/bmjquality.u201303.w827

8. Bethune R, Campbell K, Rose A, Wassall R, Price C, Siese T, Finn R, Whitaker S. Improving weekend handover between junior doctors on medical and surgical wards. BMJ Quality Improvement Reports 2014; u483.w1045 doi: 10.1136/bmjquality.u483.w1045

9. Jardine AGM, Page T et al. Bring on the weekend Improving the quality of junior doctor weekendhandover. BMJ Quality Improvement Reports 2014; u202379.w1297 doi:10.1136/bmjquality.u202379.w1297

10. Raptis DA, Fernandes C, Chua W, Boulos PB. Electronic software significantly improves quality of handover in a London teaching hospital. Health Informatics J. 2009 Sep;15(3):191-8. doi: 10.1177/1460458209337431.

11. Govier $M$, Medcalf $P$. Living for the weekend: electronic documentation improves patient handover. Clin Med. 2012 Apr;12(2):124-7.

12. Palmer E, Richardson E, Newcombe H, Borg C. The F.R.I.D.A.Y.S. checklist - Preparing our patients for a safe weekend. BMJ Quality Improvement Reports 2013; u660.w502 doi:10.1136/bmjquality.u660.w502

\section{Declaration of interests}

None

\section{Acknowledgements}

Caroline Culwick, Christopher Devine and Catherine Coombs all contributed equally to this project.

We would like to acknowledge Dr Steve Ramcharitar, consultant cardiologist at GWH, Ms Susan Chalstrey, consultant ENT surgeon at GWH, and Mr Chris Thorn, consultant general and colorectal surgeon at $\mathrm{GWH}$, for their help and advice during this project. 